\title{
Soil-Transmitted Helminths in the USA: a Review of Five Common Parasites and Future Directions for Avenues of Enhanced Epidemiologic Inquiry
}

\author{
Mary K. Lynn ${ }^{1}$ (D) Josephine A. Morrissey ${ }^{1} \cdot$ Donaldson F. Conserve ${ }^{2}$ \\ Accepted: 20 November 2020 / Published online: 30 January 2021 \\ (C) The Author(s), under exclusive licence to Springer Nature Switzerland AG part of Springer Nature 2021
}

\begin{abstract}
Purpose of Review The aim of this review is to present a comprehensive look at five intestinal soil-transmitted helminths (STHs) of global health importance that may continue to plague low-income and rural areas of the USA and argue the need for enhanced surveillance of these infections, which primarily affect the nation's most vulnerable groups.

Recent Findings Human STH infections in middle- and high-income countries are at particular risk for being undiagnosed; as common symptoms are non-specific, differentials require a high index of clinical suspicion, and cases are concentrated in areas of poverty where access to care is limited. Although autochthonous STHs are thought to be rare in the USA, infections were once common in the American South and Appalachia and robust epidemiologic surveillance is limited post 1980s. However, recent community studies and case reports from small-scale farms and areas of high rural or inner-city poverty reveal the potential for persisting helminth infections in distinct populations of the country.

Summary STHs are among the most common neglected tropical diseases globally causing significant morbidity in underserved communities and contributing to the continuation of cycles of poverty within vulnerable populations. Due to possible severe disease sequalae and their ability to perpetuate poverty and poverty-associated health outcomes within already vulnerable groups, it is vital that surveillance for these infections is enhanced to bolster health equity in the USA.
\end{abstract}

Keywords Helminths · STH · Soil-transmitted helminths $\cdot$ USA $\cdot$ Autochthonous

\section{Introduction}

Soil-transmitted helminths (STHs) are among the leading neglected tropical infections globally, disproportionately affecting children in the world's most underserved communities [1]. After decades of mass drug administration (MDA), the Global Burden of Disease Study 2017 revealed significant

This article is part of the Topical Collection on Emerging Vector Borne Diseases in the U.S.

Mary K. Lynn

lynnmk@mailbox.sc.edu

1 Laboratory of Vector-Borne and Zoonotic Diseases, Department of Epidemiology and Biostatistics, Arnold School of Public Health, University of South Carolina, Columbia, SC, USA

2 Department of Health Promotion, Education, and Behavior, Arnold School of Public Health, University of South Carolina, Columbia, SC, USA reductions in disease prevalence on a global scale [1]. However, in the USA where MDA is not suggested, a paucity of research has existed in this arena for nearly 40 years, despite an extensive documented history of helminth infections in the American South and Appalachia where rates of hookworm infection have previously reached $40 \%$ prior to the 1980s [2]. Furthermore, the USA income gap has risen in recent decades, along with $\$ 2$-a-day poverty among children $[3,4]$. This widening gap increases the potential for breakdown of community infrastructures and home sanitation systems. Suitable environmental conditions for nematode viability in this region, concentrated areas of child poverty that exceed national averages, and pockets of inadequate plumbing facilities suggest that these infections may continue to plague children and adults in certain vulnerable communities of the USA with an array of clinical outcomes ranging from impaired cognition to blindness and death in the most extreme cases [5].

A group of parasitic worm species, Ascaris lumbricoides (roundworm), Trichuris trichiura (whipworm), and Necator 
americanus/Ancylostoma duodenale (hookworm), are the 3 most common soil-transmitted helminths in man. Strongyloides stercoralis (strongyloidiasis) and Toxocara spp. (toxocariasis) are two additional STHs that have important veterinary and human health impacts and are considered neglected tropical infections of poverty in the USA. Children are particularly susceptible to STH infection, and when left untreated, lifelong consequences can occur, including malnutrition, iron deficiency, stunting, and delayed cognitive development [6]. Test scores, attendance, and other school achievement milestones can be impacted by these infections leading to lower wage earning in adulthood [6]. Though subclinical disease is common in children, complications particularly in the immunocompromised can lead to potentially fatal conditions including intestinal obstruction, visceral larva migrans, blindness, disseminated disease, respiratory failure and hemorrhage [6-10]. Pregnant women and adults with comorbidities such as HIV are additionally susceptible to severe outcomes and death in extreme cases [11].

National initiatives in the USA have claimed elimination of ongoing human transmission cycles in the formerly endemic American South; however, satisfactory socioeconomic and environmental conditions present the potential for persistence of such infections, especially in areas of poverty with poor sanitation and hygiene $[10,12]$. Estimates based on the American Community Survey 2017 suggest a possible 1.2 million Americans without access to adequate plumbing [13••]. Only Texas lists STHs as a mandatory reportable condition, although this recent addition does not publicly report infection origin [14]. A decades-long lack of active surveillance and mandatory reporting allows for the potential of an undiagnosed re-emergence $[14,15]$. Furthermore, the nonspecific nature of clinical illness, the commonality of subclinical disease, and the general lack of clinical suspicion create a scenario in which incidence and prevalence of current infection in the USA are largely unknown and likely underdiagnosed [16]. We aim to present a comprehensive look at five intestinal parasite infections of global health importance that may be persisting in low-income and rural areas of the USA and argue the need for enhanced focalized surveillance of these infections, which primarily affect the nation's most vulnerable groups.

\section{Five STHs of Public Health Importance}

\section{Ascaris lumbricoides and $A$. suum}

Ascariasis affects up to 1.2 billion individuals globally, and is one of the most common neglected tropical diseases, though manifestations of infection in humans typically present subclinically $[17,18]$. Two documented species of Ascaris predominate, A. lumbricoides in humans and A. suum in swine; however, the reported ranges of these nematodes overlap, indicating both species infect both humans and swine [18]. In high-income countries, many indigenous infections are thought to originate from zoonotic A. suum [19]. Incident zoonotic transmission can occur within other mammalian species including horses, racoons, dogs, cows, and marine mammals [20]. Human and animal transmission primarily ensues through the fecal oral route when embryonated eggs are ingested from contaminated soil or feces. Larvae then hatch and migrate through the intestinal tract to the lungs where maturation and dissemination occur through the bronchial tree. Mature larvae then migrate back to the intestine to develop into adults [21]. Adult female worms in the intestines produce approximately 200,000 eggs per day [22], and adult worms can survive in human host systems up to 2 years. In soils, fertilized eggs can persist up to 10 years [15, 22, 23]. Manifestations of ascariasis include impaired cognition, vitamin A deficiency, stunting, and asthma [6]. Complications can occur in those infected with intestinal obstruction or when rogue worms invade other host systems including the liver, pancreas, or lungs [23].

\section{Necator americanus and Ancylostoma duodenale}

Necator americanus and Ancylostoma duodenale are the primary nematode species responsible for hookworm, affecting 430 million people globally [24]. Transmission occurs through direct skin penetration, typically when walking in bare feet in areas with poor sanitation and infrastructure [9]. Filariform larvae travel from blood vessels to the bronchial tree, are then swallowed, and develop into adults in the small intestine where they may live for several years [9]. These nematodes thrive in warm sandy or loamy soils with adequate moisture, and have been associated with mining industries and the southern USA [2]. Clinical symptoms typically include reduced cognition, anemia, stunting, and protein loss [6]. Of the STHs, hookworm accounts for the greatest years lived with disability and substantial economic burdens [24].

\section{Trichuris trichiura}

Trichuris trichiura is the etiologic agent for human whipworm disease, another STH of major global health concern. Like other STHs, whipworm is linked to poverty and areas of poor sanitation, and primarily occurs in underserved communities [25]. This nematode causes infection through the fecal oral route with ingestion of infective embryonated eggs, after which maturation occurs to adult worms in the colon [25]. Adult female worms may shed up to 20,000 eggs per day and survive up to 1 year in the human host and multiple years in soils. Though many children remain asymptomatic, infection can lead to growth stunting in children, colitis, inflammatory bowel disease, and in extreme cases, trichuris dysentery 
syndrome $[6,25]$. Prevention is the best method, as traditional antihelminthics have poor treatment efficacy and reinfection has been documented [26].

\section{Strongyloides stercoralis}

Strongyloidiasis occurs through skin penetration of the infective stage filariform larvae, and like other helminths can be found in warm regions of the world with high humidity [8, 27]. S. stercoralis is typically found in rural areas with limited sanitation. It is estimated up to 100 million are infected globally with infection most often occurring in childhood from infected environmental sources [8]. Infection can last decades in humans and immunosuppressed hosts can have severe clinical outcomes particularly when hyperinfection occurs and worms are disseminated to extraintestinal organs like the lungs, liver, heart, and central nervous system [27, 28]. When severe infection occurs, manifestations include intestinal obstruction, hemorrhages, and heart and respiratory failure leading to a $90 \%$ mortality rate in untreated disseminated infection [29]. In the southeastern USA, mortality rates from this infection are highest among Caucasian men with immunocompromising comorbidities. Demographics of strongyloidiasis mortality rates differ across region of the USA, though regional differences in race and ethnicity are not fully understood $[30,31]$. S. stercoralis is unique due to its autoinfective mechanism leading to decades-long infection, and it is particularly difficult to diagnose, as variation in ova shedding in stool leads to low sensitivity of stool microscopy for this helminth $[10,11,17]$. Furthermore, sexual contact is believed to be a significant route of transmission for $S$. stercoralis in the USA particularly with men who have sex with men (MSM) [11].

\section{Toxocara canis and T. cati}

Toxocariasis is a neglected infection with Toxocara canis (canine host) or T. catis (feline host) ova that can cause cutaneous larva migrans and impaired cognitive function, particularly in children [32]. This often occurs through poor hand hygiene after contact with contaminated environments, unwashed produce, or undercooked meats [33]. Contaminated sandboxes, feline litter boxes, and other high-contact environments play an important role in local transmission foci. This nematode does not mature to adulthood in human hosts, but can live in human organs and tissues for approximately 5 years [34]. Though largely asymptomatic, those experiencing clinical manifestations typically present with visceral or ocular larva migrans [23, 32]. Visceral infection may result in clinical features including anorexia, anemia, fever, liver inflammation, abdominal pain, and meningoencephalitis. Ocular cases of disease can result in vision loss and granuloma, and are not uncommon across the southern USA [35].
What Is Known About STH in the USA?: Past and Present

Clinical and epidemiologic research efforts became focused on treating for and characterizing the burden of hookworm in the USA in the early twentieth century with $\$ 1$ million in funding donated by the Rockefeller Sanitary Commission in their efforts to eliminate "the germ of laziness" from the American South [10]. Through establishment of public dispensaries, major efforts were focused into educating the public, screening, and providing treatment across the southern states. State surveys found prevalence of hookworm up to $76 \%$ in Alabama, $53.4 \%$ in South Carolina and $65.7 \%$ in Georgia $[2,24]$. Other historic surveillance studies found highly variable rates of infection from 0 up to $14.8 \%$ in a school-based study of children 10 to 14 years in Kentucky. Another focusing on rural underserved schools in Tennessee found $19.6 \%$ infection prevalence [10]. Regional efforts identified nearly $40 \%$ prevalence of hookworm in the total population of 11 southern states surveyed [2].

While the Rockefeller campaign mainly aimed to combat hookworm infection, screening programs revealed burdens of other STHs across the southern states. Ascariasis infection prevalence ranged from $1.3 \%$ in Georgia Caucasian children ages $5-15$ to $49.4 \%$ in a second study of Cherokee Native American children in North Carolina ages 6-16 years [10]. Historic evidence of trichuriasis infection prevalence in southern states widely varies by study population. However, when focused on at-risk groups, higher rates were noted among school-based Kentucky children with $55.2 \%$ infection rate, and another school-based study of Cherokee Native American children reported a $38.0 \%$ infection rate in North Carolina [10]. S. stercoralis prevalence rates were as high as $3.8 \%$ in Kentucky school children [10].

Through these efforts coupled with improvements in infrastructure in the region, the burden of disease fell and school attendance, literacy, and the southern economy rose in areas where the campaign had significant reach [24]. After the 1980s, it was believed that hookworm had been eliminated from the south due to the efforts of the Rockefeller foundation along with improvements in sanitation and shifts towards an industry- rather than agriculture-dominated economy. However, surveillance efforts and large-scale epidemiologic studies ceased after this time period providing little evidence that elimination had truly been achieved.

Despite the great strides made in these areas, concentrated areas of poverty continue to persist in environmentally suitable conditions for nematode viability (Table 1). Human cases of ascariasis have been reported from the recent literature in isolated instances incidentally through routine colonoscopy or radiology. One such case from 2013 identified a 58-year-old African American man with comorbidities of diabetes mellitus and hypertension whose only travel history included several visits to 
Table 1 Estimated prevalence of STH in the USA with results of past and current prevalence studies, 2010-2020

\begin{tabular}{lll} 
Intestinal parasite & $\begin{array}{l}\text { Estimated prevalence } \\
\text { current }\end{array}$ & $\begin{array}{l}\text { High-quality prevalence studies } \\
\text { prior to } 1980[10]\end{array}$ \\
\hline $\begin{array}{c}\text { Ascaris lumbricoides, } \\
\text { A. suum (roundworm) }\end{array}$ & 4 million [26] & $\begin{array}{l}\text { Highest Prevalence: } 49.4 \% \text { in } 631 \\
\text { Cherokee Native School } \\
\text { children ages 6-16, NC } \\
\text { Lowest: 1.3\% in 3729 Caucasian } \\
\text { school children ages 5-15, GA }\end{array}$ \\
&
\end{tabular}

$\begin{array}{lcc}\begin{array}{l}\text { Ancylostoma duodenale, } \\ \text { Necator americanus } \\ \text { (hookworm) }\end{array} & \text { unavailable } & \text { Highest Prevalence: } 19.6 \% \text { in } \\ & & 2908 \text { School children ages } \\ & 5-16, \text { rural poverty area, TN } \\ & \text { Lowest: } 0 \% \text { in } 843 \text { individuals, } \\ & \text { random community sample, } \\ & \text { KY }[10] \\ & \\ \text { Trichuris trichiura } & \text { Hnavailable } & \\ \text { (whipworm) } & & \text { Highest: } 55.2 \% \text { in } 366 \text { Native } \\ & \text { born School children ages } \\ & 6-12, \text { KY } \\ & \text { Lowest:: } 0.5 \% \text { in } 3729 \\ & \text { Caucasian school children } \\ \text { Strongyloides stercoralis } & \text { ages } 5-15, \text { GA } \\ & 68,000-100,000[26] & \text { Highest: } 3.8 \% \text { in } 366 \text { Native } \\ & \text { born School children ages } \\ & 6-12, \text { KY } \\ & \text { Lowest: } 0 \% \text { in } 439 \text { School } \\ & \text { children ages } 10-14, \mathrm{KY}\end{array}$

Toxocara canis, T. cati
1.3-2.8 million [26] unavailable
Recent reports (last 10 years)

2018: 8-year old boy with Loeffler syndrome and no travel history from a small hog farm, LA [19]

2018: 4-month-old beef calves with respiratory illness, IA [36]

2014-2015: 31.5\% prevalence feral hogs obtained from hunters on public lands, LA [19]

2013: 58-year-old man during routine colonoscopy (no international travel history, but frequent visits to SC), NY [22]

2010-2013: 14 suspected, 8 confirmed human cases from 7 unrelated small-scale farms across 6 counties (10/14 no travel history); 5 porcine cases confirmed, ME [15]

2010:15 suspected, 1 confirmed bovine case, MO [20]

2017: $34.5 \%$ stool samples from 55 individuals in area of failing sanitation and poverty, Lowndes County, AL [24] (N. americanus)

2017: 59-year-old man with no travel history presenting with CLM, CA TX (Ancylostoma spp.) [37]

2016: 14-month-old canine with hookworm invasion of the brainstem, TX (Ancylostoma spp.) [38]

unavailable

2020: 68-year -old man with COVID-19 infection immigrating 20 years prior from Ecuador, CT

2020: $16.5 \%$ positive serology in 97 individuals from a community with failing sanitation/poverty, central TX (75\% with no travel history) [13••]

2017: 61-year old US-born man with HIV coinfection, no significant travel history, CT [11]

2017: 7.3\% stool samples from 55 individuals in area of failing sanitation and poverty, Lowndes County, AL [24]

2013: $1.9 \%$ in 378 positive serology from persons presenting to Remote Area Medical Clinics, KY (only 1 positive participant had significant travel history) [28]

2019: 3-year-old girl with OLM, previously healthy presenting with blurry vision and headache, AK [33]

2017: $5.2 \%$ positive serology from 11 individuals testing positive for any GI parasites in area of failing sanitation and poverty, Lowndes

County, AL [24]

2015-2016: 66.7\% contamination with eggs \& larvae in 10/15 soil samples from the Bronx, $38.9 \%$ in $7 / 18$ soil samples from Staten Island, $33.3 \%$ in 6/18 soil samples from Queens, $30.8 \%$ $4 / 13$ soil samples from Brooklyn, $29.6 \%$ in $8 / 27$ soil samples from Manhattan, NY [39]

2014: $4.6 \%$ egg positive fecal samples from 523,934 cats and $1.8 \%$ egg positive fecal samples from 2,750,718 dogs, national [40]

2014: 75-year-old female with pulmonary toxocariasis (only travel history 15 years prior to Caribbean), NJ [41]

2011-2014: 5.1\% overall seroprevalence from 13,509 individuals from NHANES 2011-2014, national [42]

2009-2010: 68 case reports of OLM in individuals aged 1-60, national [43] 
South Carolina from his home in the Bronx, New York [22]. Small community outbreaks related to pig husbandry have also occurred in Maine, with 14 probable ( 8 confirmed) human cases of roundworm infection from 6 counties reported between 2010 and 2013 [15]. One case of Loeffler's syndrome was identified in an 8-year-old boy on a family farm in Louisiana, where environmental sampling revealed a high amount of infective-stage eggs [19]. The source of his infection was suspected to be exposure to inadequate disposal of pig manure and open drainage systems on the property. The pig pen had a water hose that rested in the fecal runoff of the pigs, and an open septic tank with fecal runoff was located at the entrance of the pig pen. As both A. lumbricoides and $A$. suum have been documented to cause infection in humans, many of these recent case reports have occurred in agricultural areas. Infections common in commercial and feral swine [44] have also been documented in other mammalian species, like cattle in Missouri and Iowa [20,36].

Due to conducive environmental conditions in the southeastern USA, prevalence of ascariasis is reported to be high and human infection can last for years; however, subclinical infection manifestation is common and underdiagnosed [22]. Feral swine are invasive agricultural pests and the exponential population growth of these animals since the 1960s in southeastern and southwestern coastal areas of the USA could potentially contribute to the burden of ascariasis infections in areas where these populations thrive [19, 45]. Ascaris prevalence studies conducted by the Louisiana Animal Disease Diagnostics Laboratory within these feral swine populations found $31.5 \%$ prevalence in Louisiana populations [19]. Estimates predict nearly 4 million cases of ascariasis persist today in the southern part of the USA and Appalachia [26].

A 2017 study in Lowndes County, AL, an area where half of households have failing or absent sewage infrastructure, 34.5\% of area residents were hookworm positive though parasite loads were low [24]. This same study also found $7.3 \%$ S. stercoralis and 5.2\% Toxocara infection prevalence in the population [24]. In addition, cases of cutaneous larva migrans (CLM) from hookworm have also been acquired in the USA [37]. Although most of these case are associated with travel history to endemic regions of the globe, individual cases and small outbreaks from coastal states have been reported and provide continuing evidence that hookworm persists in this country [37].

In recent years, higher sensitivity and specificity molecular assays have been developed to assess $S$. stercoralis infection that may assist future surveillance efforts of this parasite worldwide [46]. The five STHs discussed in this review have been showed to affect certain racial and ethnic groups at different rates, and strongyloidiasis has been more commonly linked to Caucasian individuals living in poverty in Appalachia [26]. Above all, strongyloidiasis has been considered a disease of disadvantage, occurring at highest frequencies in those persons who are socioeconomically under the poverty line rather than in certain geographies [47]. Surveillance studies in 2013 in rural
Kentucky demonstrated $1.9 \%$ seroprevalence of S. stercoralis in individuals presenting to rural health clinics; only one individual had travel history outside of the USA [31]. A 2020 study of helminth infections from an underserved Texas community found $16.5 \%$ of screened individuals positive through serological assay [13*0]. Intersecting sociodemographic factors have led to current estimates of strongyloidiasis of up to 100,000 people in the USA [26].

The most recent high-coverage studies of STH focus on toxocariasis. Research based from over 20,000 serum samples from the National Health and Nutrition Examination Survey (NHANES) III 1988-1994 indicate the USA prevalence to be approximately $13.1 \%$, with the highest seroprevalence in nonHispanic Blacks at 21.2\% [23, 32, 48, 49]. Serologic prevalence from those in the southern USA was over $17 \%$ [32]. NHANES 2011-2014 serologic testing found 5.0\% overall seroprevalence in 13,509 persons $>6$ years of age surveyed with $10.2 \%$ prevalence in those living below the poverty line $[34,42]$. A study of this helminth in New York City boroughs from 2015 to 2016 found the highest, $66.7 \%$ contamination in soil samples obtained from the Bronx, and lowest, $29.6 \%$ soil contamination rate in Manhattan. Moreover, infective eggs were only found in the Bronx playgrounds, with over 70\% of eggs recovered in embryonic form and the highest egg burden of the study areas [39]. Only T. canis DNA was identified in this study targeting feral cats as the source of these nematodes. An extensive national veterinary study of toxocariasis in the USA revealed $4.6 \%$ prevalence in cats and $1.8 \%$ prevalence in dogs in 2014 [34]. Recent bolstered interest of ocular larva migrans, toxocariasis manifestation causing severe and lasting vision continues to occur in the USA especially in southern states [35, 50].

Human studies have consistently shown a positive association with age and Toxocara antibody detection, indicating children may not be more likely to be infected with this STH, unlike others presented in this review [34]. Furthermore, this helminth does not appear to be limited to the warmer climates of the southeast, but rather in inner-city poverty and areas with populations of stray cats and dogs or other communities with cultural practice of having freeroaming pets. However, certain racial and ethnic groups have shown to be more affected. Estimates based on the Blue Marble NTD framework suggest there may be up to 2.8 million individuals of low SES suffering from this infection, and that Black persons are disproportionately affected [26].

\section{Vulnerable Groups}

\section{Adults and Children Living Below the Poverty Line}

Table 2 shows commonly cited risk factors for STH infections; these factors intersect among poverty and inadequate 
Table 2 Major risk factors for helminth infections include poverty and underlying health conditions; however, the demographic risk factors differ between helminths. A summary table of common statistically significant associations is presented. As most cases of STH in the USA occur in immigrant populations and travelers returning from endemic areas, foreign-born persons and travel have not been included in this table for individual infections

\begin{tabular}{|c|c|c|c|}
\hline Intestinal parasite & Associated risk factors & Regions of USA & References \\
\hline $\begin{array}{l}\text { Ascaris lumbricoides, A. suum } \\
\quad \text { (roundworm) }\end{array}$ & Poverty, children, lacking sanitation \& hygiene & $\begin{array}{l}\text { Southern states, southeast, } \\
\text { Appalachia }\end{array}$ & {$[22,26,44]$} \\
\hline $\begin{array}{l}\text { Ancylostoma duodenale, } \\
\text { Necator americanus } \\
\quad \text { (hookworm) }\end{array}$ & $\begin{array}{l}\text { Extreme poverty, children, lacking sanitation \& } \\
\text { hygiene }\end{array}$ & Southern United States & {$[2,24]$} \\
\hline Trichuris trichiura (whipworm) & Poverty, children, lacking sanitation \& hygiene & Southeast, Appalachia & {$[24,26]$} \\
\hline Strongyloides stercoralis & $\begin{array}{l}\text { Caucasian ethnicity, poverty, immunocompromised, } \\
\text { HIV comorbidity, alcoholism, male sex }\end{array}$ & Appalachia & {$[26,31],[11]$} \\
\hline Toxocara canis, $T$. cati & $\begin{array}{l}\text { Black ethnicity, poverty, male sex, lead ingestion, } \\
\text { pet ownership, low educational attainment, } \\
\text { cognitive impairment, elevated lead } \\
\text { concentration, pica, nutritional status, } \\
\text { immunocompromised }\end{array}$ & $\begin{array}{l}\text { Southern states, } \\
\text { Appalachia, } \\
\text { inner cities }\end{array}$ & {$[6,26,32,33,39,42,50-52]$} \\
\hline
\end{tabular}

plumbing, primarily in the southern USA. Lower life expectancy, child mortality, and higher risk for heart disease and other chronic conditions have been linked to poverty [17]. The gap in life expectancy between the top and bottom $1 \%$ of earners in the USA has widened in the last 50 years, with a difference of 10.1 years for women and 14.6 years for men as of 2017 [53]. As populations falling into these risk categories tend to live in deep or rural poverty, gastrointestinal nematode infections may contribute to the cycles of poverty experienced in these areas due to disability-adjusted life years and educational attainment [32]. Many of the most commonly cited risk factors for developing STH infection stem out of deep poverty, particularly when experienced during childhood and when further hindered by helminth infections, forming a positive feedback loop breeding further poverty. Figure 1 illustrates that STH may be proverbial at the tip of the iceberg, as multiple health disparity factors culminate in heightened opportunities for STH infection.

Since transmission mostly occurs either through the fecaloral route via ingestion of infective ova, or through direct skin contact with larvae, STHs are commonly considered infections of childhood. Children's tendency to interact with outdoor environments at home or school, and lacking hygiene practices increase exposure risk for this age group [6]. For example, some STH infections like toxocariasis have been associated with exposure to infected sandboxes and playgrounds $[33,34]$. Though the majority of children will show mild or no clinical symptoms, some of these infections may persist for years in the body and contribute to lower test scores and cognitive impairment [50]. Maximum parasite burden with trichuriasis and ascariasis nematodes typically manifest in primary school years, while hookworm load typically peaks in secondary school or young adulthood [6]. Cognitive delays, low school attendance, and performance of infected children may limit future opportunities for higher education and career paths which further exacerbates poverty cycles. Despite improvements in sanitation and infrastructure on a national level, in the USA, 1.7 million households and 3.6 million children are subsisting on less than two dollars per day, with southeastern states having a disproportionate burden of impoverished populations [49]. A 2015 USA poverty study uncovered a rising number of children living at $150 \%$ or below of the poverty line [3].

Further findings uncovered that low-income children living below the poverty line were less likely to be non-Hispanic Caucasian and were regionally concentrated in the southeast and Appalachia suggesting that pockets of poverty are not demographically homogenous [3]. Individuals living in rural areas of the USA and those living under the poverty level are less likely to have health care access and thus present a challenge for identification of cases and the need for surveillance studies to prevent children in these underserved areas from further negative health outcomes $[31,53]$. Health care access is a particular challenge in rural areas where physician shortages continue to occur [53]. The relationship between poverty and poor health status, geographic, and financial constraints on health care access may make individuals in these circumstances at higher risk for infections including STHs in areas where they may continue to exist.

\section{Adults and Children Lacking Access to Adequate Plumbing}

Lack of access to functioning sanitation systems and plumbing continues in the USA despite the relative wealth of the nation compared to certain low- and middle-income countries, and the progress made since the Rockefeller undertaking. The US Census Bureau estimates nearly half a million occupied housing units in the USA lacked complete plumbing facilities translating to 1.2 million individuals, and $18.1 \%$ of homes are not hooked to a public sewer [13••]. A 2019 study of water and plumbing access in the country found overlapping spatial 
Fig. 1 Illustrates root causes of $\mathrm{STH}$, the greater cycles of poverty, and associated healthrelated risk factors providing conducive conditions for these infections to persist in the USA

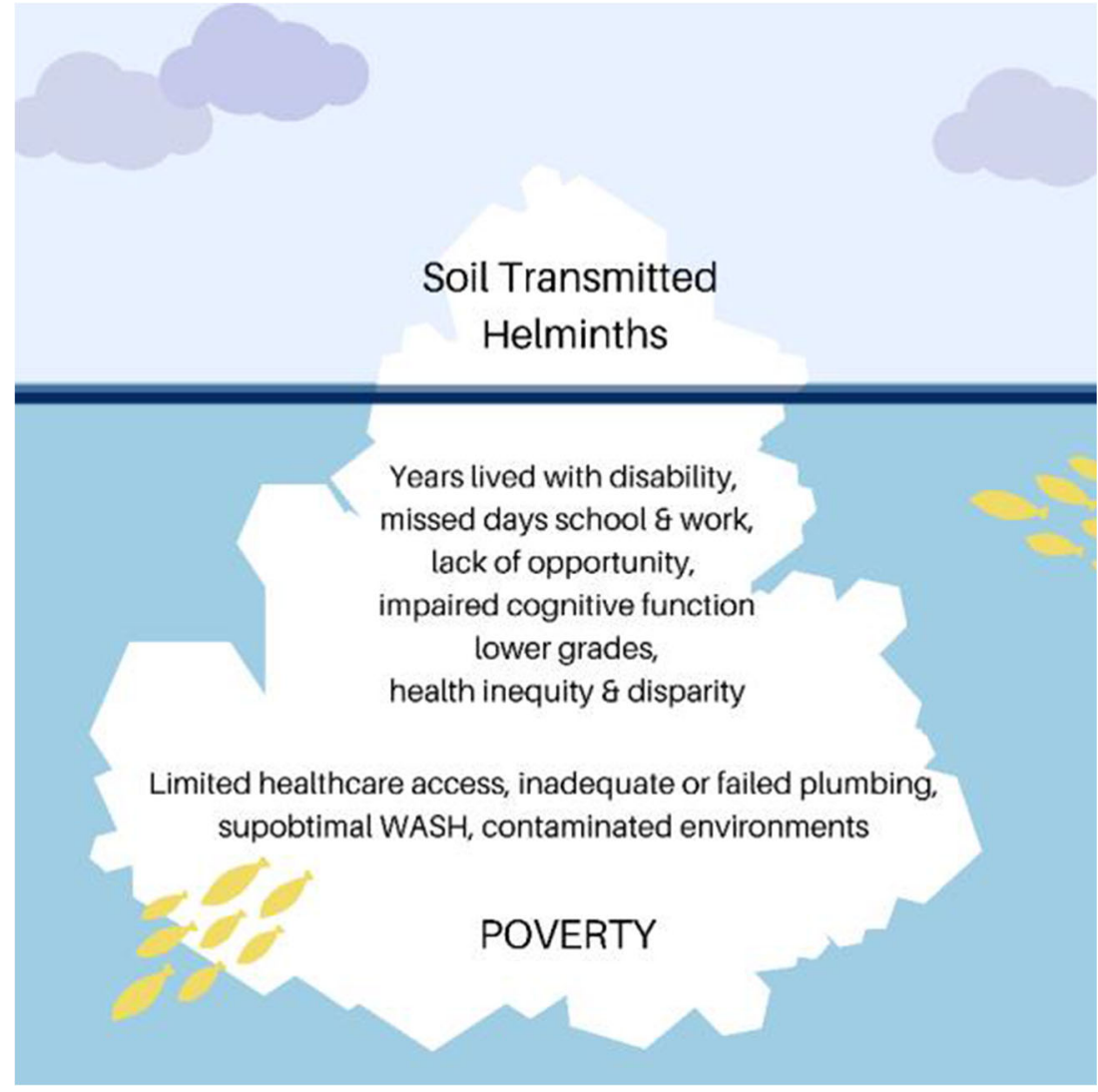

and demographic characteristics of households that were "plumbing poor" [54]. This study identified $16.6 \%$ and $16.7 \%$ of Black and Hispanic households in the nation, respectively, lack complete plumbing facilities [54]. Though American Indians and Alaska Natives account for only $1.5 \%$ of the population, $6.2 \%$ of these households lack complete plumbing. In addition, American Indians and Alaska Natives were 3.7 times more likely to lack complete plumbing than non-Hispanic Caucasians, and Black households were 1.2 times more likely to lack complete plumbing facilities particularly in the southern USA [54]. Further complicating the issue of inadequate infrastructure, in underserved communities, plumbing upkeep and septic tank maintenance may pose too great a financial burden to maintain sanitary conditions as identified in Lowndes County, AL [24].

\section{Immunocompromised}

STH infections often present asymptomatically, contributing to a limited incidence and prevalence knowledge in the USA. However, STH infections may progress to more severe clinical disease in a small number of those infected, particularly in persons who are immunocompromised [47]. S. stercoralis is thought to also be underdiagnosed in individuals living with HIV, as suggested from a case report from 2016 in a USAborn man with no significant travel history [11]. HIV- coinfected individuals have been identified as a high-risk group for this infection, making them particularly vulnerable to severe forms of disease including hyperinfection, which has a relatively poor prognosis [11]. Those undergoing HIV treatment or treatment for other chronic infections may bear a large financial cost for medication or visits to the clinic. In order to protect these vulnerable and immunosuppressed groups, clinicians should be made aware that intestinal parasites circulate undetected [11].

\section{Recent USA Immigrants}

In the USA, the majority of STH infections are thought to be imported from tropical and subtropical regions where disease burdens are high [37]. A 2020 study of recent Chicago immigrants showed $6.4 \%$ and $4.0 \%$ seroprevalence for Toxocara and Strongyloides stercoralis [55]. A Boston study of refugees and asylum seekers in the USA from 2012 to 2017 found $0.2 \%$ hookworm infection and $1.9 \%$ S. stercoralis infection, [56]. Though the US Centers for Disease Control and Prevention has established protocols for testing and treating refugee populations in the USA, there are no surveillance protocols currently in place to address these infections in documented or undocumented immigrants [55]. The northern USA is not considered to be an endemic area for parasitic helminths due to insufficient climatic conditions; however, environmental samples have shown high rates of Toxocara ova [39]. Based on historical patterns of 
immigration flow into large cities and cramped areas with insufficient infrastructure, USA immigrant populations may be at risk for STHs when living in lower income areas of large cities like NYC [55].The combination of high occupancy crowding, poor housing standards, and lack of health care access among immigrants could lead to STH familial transmission cycles. Though this review aims to focus on US-acquired infection, USA immigrants are another vulnerable group that may be at heightened risk for infection whether from lack of screening and treatment upon entering the USA or through acquisition in USA communities in which they live. Immigrants to the USA are considered duel risk for STH infection from their home countries or from the USA, and the risk exists for continuation of transmission cycles within familial clusters or neighborhoods with large foreign-born populations [55]. Due to the tendency for increased Th2 and regulatory $\mathrm{T}$ cell response elicited by intestinal parasitic infections, recent immigrants may be at higher risk for other serious infections including tuberculosis, which may have severe consequences in crowded areas of poverty [57]. Physicians should include STH in differential diagnoses in these subpopulations.

\section{Other Risk Factors}

Environmental conditions particularly in the southern USA where humidity and ambient temperature are temperate enough for nematode ova to persist in soil for years provide the opportunity for spatial targeting of high-risk regions [24]. Enzootic factors in communities with large populations of feral cats and dogs, non-commercial farming, and regions of the USA with large populations of feral swine further provide conducive conditions for these nematode transmission cycles to continue in enzootic pathways $[19,32]$. In addition to small-scale farming, pigs and feral swine, feral dogs and cats, and free-roaming pets have also been known to spread infections especially in urban parks and playgrounds via fecal droppings.

Most STHs are viable in areas of high humidity and temperature, and many areas of the southern USA maintain these climatic conditions for development and proliferation of helminths. Furthermore, Ascaris lumbricoides and Toxocara spp. can remain viable in soils even through extreme temperatures for years [58 ]. A. lumbricoides and T. trichiura viability ranges from $5-38{ }^{\circ} \mathrm{C}$ and hookworm development is suitable between approximately 8 and $45^{\circ} \mathrm{C}$ [58•]. Strongyloides stercoralis larvae are limited in lifespan outside a host organism, surviving in high humidity environments between 20 and $28^{\circ} \mathrm{C}$, and survival in the environment is typically less than 3 weeks [59].

Since December 2019, the pandemic of SARS CoV-2 has significantly impacted individuals and health care systems on a global scale. One case of disseminated strongyloidiasis with COVID-19 coinfection has been documented, highlighting the need for further investigative efforts to understand the impact of STH COVID-19 coinfections [60••]. Immunomodulating treatment to combat the virus could elicit severe reactions in those with underlying STH infections, particularly in those with preexisting comorbidities. Clinicians should consider STH infection in high-risk populations in decision-making for COVID-19infected patients.

Given the current knowledge of individualized factors that could contribute to STH infections in this country, it is vital that future interdisciplinary methodologies be employed to spatially identify areas of overlapping environmental and socioeconomic risk parameters to target high-risk zip codes and block groups within communities for surveys of human infection and intervention efforts. Current and historical research has been focused mainly in the southeastern states where STH infections were previously high. However, future research should also target areas of the southwest where access to running water and plumbing facilities are vastly limited.

\section{Gaps in the Literature}

After the 1980s, high-powered studies of STH are lacking. The vast majority of STH infection studies in the past 10 years focus primarily on recent immigrants to the USA, leaving limited evidence for STH persistence or elimination in this country. Despite disease burden estimates reaching one million, case reports and surveillance studies are limited. Keywords used to obtain highquality studies were entered into PubMed including: "helminth, STH, soil, Ascaris, Trichuris, Necator, Ancylostoma, Strongyloides, Toxocara, hookworm, whipworm, roundworm, US, USA, United States, children, immunocompromised" yielding $<5$ results typically with the highest number of results from search terms "soil + helminth + USA" with 231 results. However, even in this case, relevant articles including case reports of persons with indigenously acquired infections remained slim and most results were not relevant to the review topic as they did not assess infection prevalence or did not occur indigenously in the USA.

\section{Future Directions}

Though the high-risk categories addressed are based on individualized factors that increase risk of acquiring STH, most of these factors stem from poverty. Similar to other neglected tropical diseases, the most impoverished communities suffer the highest burdens. Rural poverty in particular may decrease the likelihood of both health care access and septic tank maintenance.

Based on historical knowledge and limited recent studies of risk factors for STH infections in the USA, overlapping conditions like poverty, lack of complete plumbing or poor sanitation, and conducive ecological conditions present the potential that STHs persist in certain regions of the USA. Subclinical presentation and lack of health care access create challenges to identifying and diagnosing these infections. Future high-powered studies should be conducted in regions of the USA with these overlapping sociodemographic and ecological conditions to identify the veritable helminth infection burden in this country. With 
known risk factors and the ability to pinpoint areas of high risk for these infections in the country, targeted surveillance efforts should be enacted to monitor the current prevalence of STH in the USA. Physician awareness surrounding these diseases, particularly pediatricians and those serving rural health clinics and vulnerable populations, should also be heightened as neglected tropical diseases do not solely exist outside of the USA. Identifying areas where active transmission cycles are still occurring is the first step to improving equal opportunity for children and underrepresented groups living in poverty in this country. Decreasing STH infections will likely increase the economic potential and health equity of the vulnerable communities in which cycles of transmission are occurring.

Acknowledgments The authors would like to thank Kyndall DyeBraumuller for her editorial expertise and Dr. Melissa Nolan for her assistance with the conceptualization of this study.

Author Contributions M. K. Lynn: conceptualization, investigation, manuscript preparation, and visualization. Josephine A. Morrissey: investigation and manuscript preparation. Donald F. Conserve: conceptualization, methodology, review \& editing, supervision, and validation.

Data Availability Not applicable

\section{Compliance with Ethical Standards}

Conflict of Interest The authors declare that they have no conflict of interest.

Human and Animal Rights and Informed Consent This article does not contain any studies with human or animal subjects performed by any of the authors.

Ethics Approval Not applicable.

Consent to Participate Not applicable.

Consent for Publication The authors agree to the publication of this manuscript and attest that this manuscript has not been submitted elsewhere.

Code Availability Not applicable.

\section{References}

Papers of particular interest, published recently, have been highlighted as:

- Of importance

- Of major importance

1. Global, regional, and national incidence, prevalence, and years lived with disability for 354 diseases and injuries for 195 countries and territories, 1990-2017: a systematic analysis for the Global Burden of Disease Study 2017. Lancet. 2018;392(10159):1789858. https://doi.org/10.1016/s0140-6736(18)32279-7.
2. Elman C, McGuire RA, Wittman B. Extending public health: the Rockefeller Sanitary Commission and hookworm in the American South. Am J Public Health. 2014;104(1):47-58. https://doi.org/10. 2105/ajph.2013.301472.

3. Shaefer HL, Edin K, Talbert E. Understanding the dynamics of \$2a-day poverty in the United States. RSF. 2015;1(1):120-38. https:// doi.org/10.7758/rsf.2015.1.1.07.

4. "Trends in income and wealth inequality." Pew Research Center, Washington, D.C. 2020 https://www.pewsocialtrends.org/2020/01/ 09/trends-in-income-and-wealth-inequality/.

5. Hotez PJ. Neglected diseases and poverty in "The Other America": the greatest health disparity in the United States? PLoS Negl Trop Dis. 2007;1(3):e149.

6. Weatherhead JE, Hotez PJ, Mejia R. The global state of helminth control and elimination in children. Pediatr Clin N Am. 2017;64(4): 867-77. https://doi.org/10.1016/j.pcl.2017.03.005.

7. CDC. DPDx Toxocariasis Biology. 2019. https://www.cdc.gov/ dpdx/toxocariasis/. Accessed: May 5, 2020.

8. CDC. DPDx Strongyloidiasis. 2019. https://www.cdc.gov/dpdx/ strongyloidiasis/. Accessed: May 10, 2020.

9. CDC. DPDx Hookworm (Intestinal). 2019. https://www.cdc.gov/ dpdx/hookworm/. Accessed: May 5, 2020.

10. Starr MC, Montgomery SP. Soil-transmitted helminthiasis in the United States: a systematic review-1940-2010. Am J Trop Med Hyg. 2011;85(4):680-4.

11. Vazquez Guillamet LJ, Saul Z, Miljkovich G, Vilchez GA, Mendonca N, Gourineni V, et al. Strongyloides stercoralis infection among human immunodeficiency virus (HIV)-infected patients in the United States of America: a case report and review of literature. Am J Case Rep. 2017;18:339-46. https://doi.org/10. 12659/ajcr.902626.

12. Blum AJ, Hotez PJ. Global "worming": climate change and its projected general impact on human helminth infections. PLoS Negl Trop Dis. 2018;12(7):e0006370.

13.• Singer R, Xu TH, LNS H, Villar MJ, Faust KM, Hotez PJ, et al. Prevalence of intestinal parasites in a low-income Texas community. Am J Trop Med Hyg. 2020;102(6):1386-95. https://doi.org/10. 4269/ajtmh.19-0915 This manuscript identifies a low-income Texas community with serological evidence of STH infection and provides evidence for enhanced investigation of these infections in similar communities across the USA.

14. Texas Department of State Health Services. Reported case counts for selected neglected tropical diseases in Texas, 2016*-2017; 2019. https://www.dshs.texas.gov/IDCU/disease/Data.aspx. Accessed July 29, 2020.

15. Miller LA, Colby K, Manning SE, Hoenig D, McEvoy E, Montgomery S, et al. Ascariasis in humans and pigs on smallscale farms, Maine, USA, 2010-2013. Emerg Infect Dis. 2015;21(2):332-4.

16. O'Connell EM, Nutman TB. Molecular diagnostics for soiltransmitted helminths. Am J Trop Med Hyg. 2016;95(3):508-13. https://doi.org/10.4269/ajtmh.16-0266.

17. Hotez PJ. Neglected infections of poverty in the United States of America. PLoS Negl Trop Dis. 2008;2(6):e256. https://doi.org/10. 1371/journal.pntd.0000256.

18. Mathison BA, Pritt BS. A systematic overview of zoonotic helminth infections in North America. Lab Med. 2018;49(4):e61-93. https://doi.org/10.1093/labmed/lmy029.

19. Avery RH, Wall LA, Verhoeve VI, Gipson KS, Malone JB. Molecular confirmation of Ascaris suum: further investigation into the zoonotic origin of infection in an 8-year-old boy with Loeffler syndrom. Vector Borne Zoonotic Dis. 2018;18:638-40.

20. Taylor HL, Spagnoli ST, Calcutt MJ, Kim DY. Aberrant Ascaris suum nematode infection in cattle, Missouri, USA. Emerg Infect Dis. 2016;22(2):339-40. 
21. CDC. DPDx Ascariasis. 2019. https://www.cdc.gov/dpdx/ ascariasis/index.html. Accessed: May 8, 2020.

22. Kanneganti K, Makker JS, Remy P. Ascaris lumbricoides: to expect the unexpected during a routine colonoscopy. Case Rep Med. 2013;2013:1-4.

23. Woodhall D, Jones JL, Cantey PT, Wilkins PP, Montgomery SP. Neglected parasitic infections: what every family physician needs to know. Am Fam Physician. 2014;89(10):803-11.

24. McKenna ML, McAtee S, Bryan PE, Jeun R, Ward T, Kraus J, et al. Human intestinal parasite burden and poor sanitation in rural Alabama. Am J Trop Med Hyg. 2017;97(5):1623-8.

25. CDC. DPDx Trichuriasis. 2017. https://www.cdc.gov/dpdx/ trichuriasis/index.html. Accessed: May 5, 2020.

26. Hotez PJ. Blue marbel health: an innovative plan to fight diseases of the poor amid wealth: JHU Press; 2016.

27. Puthiyakunnon S, Boddu S, Li Y, Zhou X, Wang C, Li J, et al. Strongyloidiasis - an insight into its global prevalence and management. PLoS Negl Trop Dis. 2014;8(8):e3018.

28. Russell ES, Gray EB, Marshall RE, Davis S, Beaudoin A, Handali $\mathrm{S}$, et al. Prevalence of Strongyloides stercoralis antibodies among a rural Appalachian population-Kentucky, 2013. Am J Trop Med Hyg. 2014;91(5):1000-1. https://doi.org/10.4269/ajtmh.14-0310.

29. Rha B, Kelly DR, Shimamura M. Eosinophilic appendicitis attributable to Strongyloides infection in a pediatric renal transplant patient. J Pediatric Infect Dis Soc. 2012;2(3):274-7.

30. Croker C, Reporter R, Redelings M, Mascola L. Strongyloidiasisrelated deaths in the United States, 1991-2006. Am J Trop Med Hyg. 2010;83(2):422-6. https://doi.org/10.4269/ajtmh.2010.090750

31. Russell ES, Gray EB, Marshall RE, Davis S, Beaudoin A, Handali S, et al. Short report: prevalence of Strongyloides stercoralis antibodies among a rural Appalachian population-Kentucky, 2013. Am J Trop Med Hyg. 2014;91(5):1000-1.

32. Lee RM, Moore LB, Bottazzi ME, Hotez PJ. Toxocariasis in North America: a systematic review. PLoS Negl Trop Dis. 2014;8(8): e3116. https://doi.org/10.1371/journal.pntd.0003116.

33. Boye B, Wayne M, Sukumaran S, Vijayan V. Blurry vision and irregularly shaped pupil in a 3-year-old female. Clin Pediatr. 2019;58(9):1038-41. https://doi.org/10.1177/0009922819850460.

34. Bradbury RS, Hobbs CV. Chapter Sixteen - Toxocara seroprevalence in the USA and its impact for individuals and society. In: Bowman DD, editor. Advances in Parasitology: Academic Press; 2020. p. 317-39.

35. Woodhall D, Starr MC, Montgomery SP, Jones JL, Lum F, Read $\mathrm{RW}$, et al. Ocular toxocariasis: epidemiologic, anatomic, and therapeutic variations based on a survey of ophthalmic subspecialists. Ophthalmology. 2012;119(6):1211-7. https://doi.org/10.1016/j. ophtha.2011.12.013.

36. Jesudoss Chelladurai J, Derscheid R, Brewer MT. Respiratory disease associated with migrating Ascaris larvae in a beef calf. Vet Parasitol Reg Stud Reports. 2018;12:9-12. https://doi.org/10. 1016/j.vprsr.2017.12.005

37. Benedetto C, Youssef I, Shitabata P, Nami N. An Indigenous Case of Cutaneous Larva Migrans. JAOCD. 2017;38:29-30.

38. Perry A, Dangoudoudiyam S, Bolling M, Rodrigues-Hoffmann A. Aberrant Ancylostoma sp. in the brain of a dog. Vet Parasitol. 2016;223:210-3

39. Tyungu D, McCormick D, Lau C, Chang M, Murphy J, Hotez P, et al. Toxocara species environmental contamination of public spaces in New York City. PLoS Negl Trop Dis. 2020;15(5): e0008249.

40. Lucio-Forster A, Mizhquiri Barbecho JS, Mohammed HO, Kornreich BG, Bowman DD. Comparison of the prevalence of Toxocara egg shedding by pet cats and dogs in the U.S.A., 20112014. Vet Parasitol. 2016;5:1-13.
41. Ranasuriya G, Mian A, Boujaoude Z, Tsigrelis C. Pulmonary toxocariasis: a case report and literature review. Infection. 2014;42(3):575-8. https://doi.org/10.1007/s15010-014-0587-3.

42. Farmer A, Beltran T, Choi YS. Prevalence of Toxocara species infection in the U.S.: results from the National Health and Nutrition Examination Survey, 2011-2014. PLoS Negl Trop Dis. 2017;11(7):e0005818.

43. Hare AQ, Franco-Paredes C. Ocular larva migrans: a severe manifestation of an unseen epidemic. Curr Trop Med Rep. 2014;1:6973.

44. Jesudoss Chelladurai J, Murphy K, Snobl T, Bader C, West C, Thompson K, et al. Molecular epidemiology of Ascaris infecting pigs in Iowa, USA. J Infect Dis. 2016;215(1):131-8. https://doi.org/ 10.1093/infdis/jiw507.

45. McClure ML, Burdett CL, Farnsworth ML, Lutman MW, Theobald DM, Riggs PD, et al. Modeling and mapping the probability of occurrence of invasive wild pigs across the contiguous United States. PLoS One. 2015;10(8):e0133771.

46. Mejia R, Vicuña Y, Broncano N, Sandoval C, Vaca M, Chico M, et al. A novel, multi-parallel, real-time polymerase chain reaction approach for eight gastrointestinal parasites provides improved diagnostic capabilities to resource-limited at-risk populations. Am J Trop Med Hyg. 2013;88(6):1041-7.

47. Beknazarova M, Whiley H, Ross K. Strongyloidiasis: a disease of socioeconomic disadvantage. LID - https://doi.org/10.3390/ ijerph13050517 LID - E517. (1660-4601 (Electronic)).

48. Won KY, Kruszon-Moran D, Schantz PM, Jones JL. National seroprevalence and risk factors for zoonotic Toxocara spp. infection. Am J Trop Med Hyg. 2008;79(4):552-7.

49. Hotez PJ. Neglected parasitic infections and poverty in the United States. PLoS Negl Trop Dis. 2014;8(9):e3012.

50. Walsh MG, Haseeb MA. Reduced cognitive function in children with toxocariasis in a nationally representative sample of the United States. Int J Parasitol. 2012;42(13):1159-63. https://doi.org/10. 1016/j.jpara.2012.10.002.

51. Liu EW, Chastain HM, Shin SH, Wiegand RE, Kruszon-Moran D, Handali S, et al. Seroprevalence of antibodies to Toxocara species in the United States and associated risk factors, 2011-2014. Clin Infect Dis. 2018;66(2):206-2012.

52. Fan C-K, Holland CV, Loxton K, Barghouth U. Cerebral toxocariasis: silent progression to neurodegenerative disorders? Clin Microbiol Rev. 2015;28(3):663-86. https://doi.org/10.1128/ cmr.00106-14.

53. Dickman SL, Himmelstein DU, Woolhandler S. Inequality and the health-care system in the USA. Lancet. 2017;389:1431-41.

54. Deitz S, Meehan K. Plumbing poverty: mapping hot spots of racial and geographic inequality in U.S. household water insecurity. Ann Am Assoc Geogr. 2019;109(4):1092-109.

55. Herrick JA, Nordstrom M, Maloney P, Rodriguez M, Naceanceno $\mathrm{K}$, Gallo $\mathrm{G}$, et al. Parasitic infections represent a significant health threat among recent immigrants in Chicago. Parasitol Res. 2020;1: $1-10$.

56. Epstein RL, Bhagavathula M, Saag LA, Verma S, Kan CK, Mesick $\mathrm{J}$, et al. QuantiFERON-TB gold in-tube reliability for immigrants with parasitic infections, Boston. Int $\mathrm{J}$ Tuberc Lung Dis. 2019;23(4):482-90.

57. Board AR, Sazuki S. The interrelation between intestinal parasites and latent TB infections among newly resettled refugees in Texas. Int Health. 2016;8:67-72.

58. Sturrock SL, Yiannakoulias N, Sanchez AL. The geography and scale of soil-transmitted helminth infections. Curr Trop Med Rep. 2017;4(4):245-55 Factors influencing STH infection prevalence vary across scale and region of the globe. This manuscript identifies important environmental, social, and behavioral determinants of STH infection and describes the need for 
geography-specific investigation to inform targeted intervention strategies.

59. Page W, Judd JA, Bradbury RS. The unique life cycle of Strongyloides stercoralis and implications for public health action. Trop Med Infect Dis. 2018;3(2):53.

60.• Lier AJ, Tuan JJ, Davis MW, Paulson N, McManus D, Campbell S, et al. Case report: disseminated strongyloidiasis in a patient with COVID-19. Am J Trop Med Hyg. 2020;00(0):1-3. Given the rapid disease progression of COVID-19 and scale of the pandemic, it is vital that clinicians consider helminth co-infection in high- risk groups to mitigate potential negative outcomes of immunosuppresive therapy for the virus. This manuscript highlights severe complications that may occur in those with undiagnosed STH coinfections, and the critical nature of bolstered surveillance and physician awareness of STHs in the USA.

Publisher's Note Springer Nature remains neutral with regard to jurisdictional claims in published maps and institutional affiliations. 